NASA Technical Memorandum 83258

NASA-TM-83258 19820010992

\title{
EFFECT OF TACTILE VIBRATION ON ANNOYANCE TO SYNTHESIZED PROPFAN NOISE
}

Sherman A. Clevenson

DECEMBER 1981

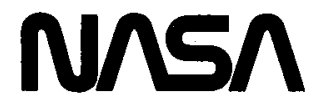

National Aeronautics and

Space Administration

Langley Research Center Hampton, Virginia 23665 



\section{SUMMARY}

A research program at NASA-Langley Research Center is being conducted to provide design information that maximizes passenger comfort for proposed propfan aircraft. Particular emphasis in this study is being placed on predicting noise and vibration environments and the resultant passenger acceptability. Previous ride quality research of this program has indicated that vibrations of sufficient intensity to produce whole body movements (at frequencies less than $30 \mathrm{~Hz}$ ) cause passenger discomfort and annoyance. Within this complex interior environment, this type of vibration interacts additively with noise to produce discomfort. However, recent questions have arisen concerning the effect of high frequency tactile vibration (i.e. greater than $30 \mathrm{~Hz}$ ) on passenger reactions. The current study addressed this question through obtaining passenger reactions to a wide range of noise with and without tactile vibration. The investigation was conducted in the passenger ride quality simulator located at the NASA-Langley Research Center using 96 subjects who evaluated either synthesized propfan aircraft noises only, or these noises combined with tactile (seat-arm) vibration. The noises ranging from $69-85 \mathrm{~dB}(\mathrm{~A})$ consisted of a turbulent boundary layer noise with a factorial combination of five blade passage frequencies $(50-200 \mathrm{~Hz})$, two harmonic rolloffs, and three tone/noise ratios. The results indicate that passenger reaction (annoyance) to noise is not significantly changed in the presence of tactile vibration.

\section{INTRODUCTION}

A research program at NASA Langley Research Center is being conducted to provide design information for passenger comfort for proposed propfan aircraft. Particular emphasis in this study is being placed on predicting noise and vibration environments and the resultant passenger acceptability. Previous ride quality research has indicated that vibrations of sufficient intensity to produce 
whole body movements (at frequencies less than $30 \mathrm{~Hz}$ ) cause passenger discomfort and annoyance. This type of vibration also has been found to interact additively with noise to produce additional discomfort. However, questions have arisen concerning the effect of high frequency tactile vibration (i.e. greater than 30 $\mathrm{Hz}$ ) on passenger reactions. Specifically, is the passenger more or less annoyed when subjected to high frequency tactile (seat-arm) vibration while being exposed to the interior noise environment? The objective of the current study is to provide an answer to the above question.

The study addressed the question through obtaining passenger annoyance responses (96 subjects) to a wide range of noises and noise spectra with and without tactile vibration, namely, vibration of the armrests. The study was conducted in a unique facility at the Langley Research Center, the Passenger Ride Quality Apparatus (PRQA). The noises, ranging in level from 69 to $85 \mathrm{~dB}(\mathrm{~A})$, consisted of a simulated turbulent boundary layer noise in combination with synthesized propfan noise tone components. Tactile vibration was provided to half of the subjects. The results of this investigation to determine the effect of tactile vibration on annoyance to synthesized propfan noise is reported herein.

\section{METHOD}

The objective of this investigation was achieved by exposing subjects to selected combinations of noise with and without armrest vibration. The following sections provide a review of the simulator, subject characteristics, subjective evaluations, physical noise-vibration environments, and test procedures used in the investigation.

\section{Simulator}

The facility used to generate the noise and vibration environments was the Passenger Ride Quality Apparatus (PRQA, shown in fig. 1 with the front bulkhead 
removed)(refs. 1-2). For this study, the cabin was fitted with two rows of first class seats (two abreast) with an electrodynamic shaker located beneath each set of seats (fig. 2). The primary vibration felt by the passenger subjects was through the armrests of the seats.

\section{Subjects}

A total of 96 subjects (12 males and 78 females) participated in the study. The subjects were obtained from a contractural pool and were paid for their participation. Their ages ranged from 18 to 68 years, with a medium age of 35 years and weighed from 102 to 272 pounds with a medium weight of 142 pounds. All subjects were audiometrically screened and were required to have no hearing losses greater than $20 \mathrm{~dB}$ at frequencies up to $6,000 \mathrm{~Hz}$.

\section{Subjective Evaluations}

A continuous unipolar scale, with associated numerical integers, was used by each subject to evaluate the annoyance of a test condition (fig. 3 ). The scale was anchored at zero with the words "ZERO ANNOYANCE." The anchor at the opposite end of the scale was "MAXIMUM ANNOYANCE." Thus, the scale continuum of increasing numbers was interpreted as representing increasing degrees of annoyance. The subjects were instructed to interpret the scale in an equal-interval fashion and to base their annoyance judgments upon the environment they experienced within each test condition.

\section{Physical Noise-Vibration Environments}

The interior noise simulating proposed propfan aircraft was synthesized based on the expected turbulent boundary layer noise with superimposed tones with different fundamental frequencies $\left(f_{n}\right)$, signal to noise ratios $(S / N)$, and rolloff ratios (R.0.). For this study, the experimental condition that resulted from a parametric combination of noise level, $f_{n}, S / N$, and R.0. either with or without tactile vibration was one test point. The values of $f_{n}$ were $50,80,100,125$, 
or $200 \mathrm{~Hz}$. Values of $\mathrm{S} / \mathrm{N}$ were 0,10 , or $20 \mathrm{~dB}$. Values of R.0. were 0 and $10 \mathrm{~dB}$. $S / N$ is defined as the level of the tone compared to the adjoining third octave levels. R.0. is defined as the decrease in level for the harmonics of the tone. The " $A$ " weighted sound pressure levels varied from $69-85 \mathrm{~dB}(A)$. The seat-arm vibration was measured and recorded on a current turboprop aircraft. Similar signals were synthesized (see fig. 4) and used to control the underseat shakers (fig. 2) such that the armrests' vibration was identical for every test point that had armrest vibration regardless of noise level. The vibration was essentially random with a $100 \mathrm{~Hz}$ tone with a peak level of approximately $.12 \mathrm{~g}$. Separate groups of subjects were used for the vibration and no vibration conditions.

\section{Test Procedures}

A typical day's testing consisted of instructing passenger-subjects in the use of the rating sheets and then escorting them into the cabin and exposing them to the selected stimuli over a test period of approximately 1-1/2 hours. The test period was divided into two parts with a 15-minute rest period in the middle. The tasks for each passenger-subject (four subjects concurrently) were to experience the stimuli and then to rate their annoyance on the annoyance scale described earlier. The order of presentation of stimuli was balanced and randomized. Each stimulus consisted of a 3 seconds rise time, 15 seconds of noise with or without seat-arm vibration, 3 seconds decay time, and 9 seconds of rating time.

\section{RESULTS AND DISCUSSION}

The results are shown in figures 5-8. Annoyance ratings are shown as a function of frequency, namely, the fundamental frequency of the tones, in figure 5. The annoyance ratings are the average ratings over all $S / N$ and $R .0$. values taken at the average noise level. The circles represent no tactile vibration and the squares represent tactile vibration. Although the annoyance ratings for the passenger-subjects exposed to tactile vibration are consistently lower than the 
ratings for those not exposed to tactile vibration, the small difference is of no statistical significance. There also appears to be little effect of the frequency of tones.

Annoyance ratings are shown as a function of $S / N$ ratios in figure 6 where the annoyance ratings are the average ratings over all tones and R.0. ratios taken at the average noise level. Although the ratings are consistently lower by the passenger subjects exposed to tactile vibration, the small difference is of no statistical significance. The increase in annoyance ratings as the $S / N$ ratio is increased is not unexpected.

Annoyance ratings are shown as a function of R.0. ratio in figure 7 where the ratings have been averaged over all tones and $S / N$ ratios at the average noise leve1. The ratings are consistently lower for those passenger subjects exposed to tactile vibration. However, the small difference is of no statistical significance. The slight decrease in ratings as the R.0. ratio was changed from 0 to 10 $d B$ is also of no statistical significance.

The effects of tactile vibration are indicated in figure 8 where annoyance ratings are shown as a function of " $A$ " weighted sound pressure levels. The solid line represents a regression analysis for all the annoyance ratings of passengersubjects who were not exposed to seat-arm vibration whereas the dashed line indicates the regression line for those passenger-subjects who were exposed to seat-arm vibration. Both lines indicate the usual increase in annoyance with increased noise level. The ratings at the same noise levels are lower for those passenger subjects who were exposed to tactile vibration. In order for a person to have the same annoyance response to tactile vibration as he had with no tactile vibration, the noise level would have to be increased by $1 \mathrm{~dB}$. However, this difference is not statistically significant. Thus, passenger reaction (annoyance) is not significantly changed in the presence of tactile vibration. 


\section{CONCLUDING REMARKS}

The effect of tactile vibration on annoyance to synthesized propfan noise has been investigated through obtaining passenger-subject reactions to a wide range of noise environments. The investigation was conducted in the Passenger Ride Quality Apparatus located at NASA-Langley Research Center using 96 passenger-subjects who evaluated synthesized propfan aircraft noises only, or these noises combined with tactile (seat-arm) vibration. The noises ranging from $69-85 \mathrm{~dB}(A)$ consisted of a turbulent boundary layer noise with a factorial combination of five blade passage frequencies (50-200 Hz), two harmonic rolloffs, and three tone/noise ratios.

No significant effects of blade passage frequency (tones), signal/noise or rolloff ratios were determined on annoyance response of passenger subjects whether or not they were exposed to tactile vibration. In addition, there were no significant differences with noise exposure in the annoyance responses of the passengers whether or not they were exposed to tactile vibration.

\section{REFERENCES}

1. Clevenson, S. A.; and Leatherwood, J. D.: On the Development of Passenger Ride Acceptance Criteria. Shock and Vibration Bulletin 43, pt. 3, U.S. Dept. of Defense, 1973, pp. 105-111.

2. Clevenson, S. A.; and Leatherwood, J. D.: Effect of Noise Spectra and a Listening Task upon Passenger Annoyuance in a Helicopter Interior Noise Environment. NASA TP 1590, December 1979. 


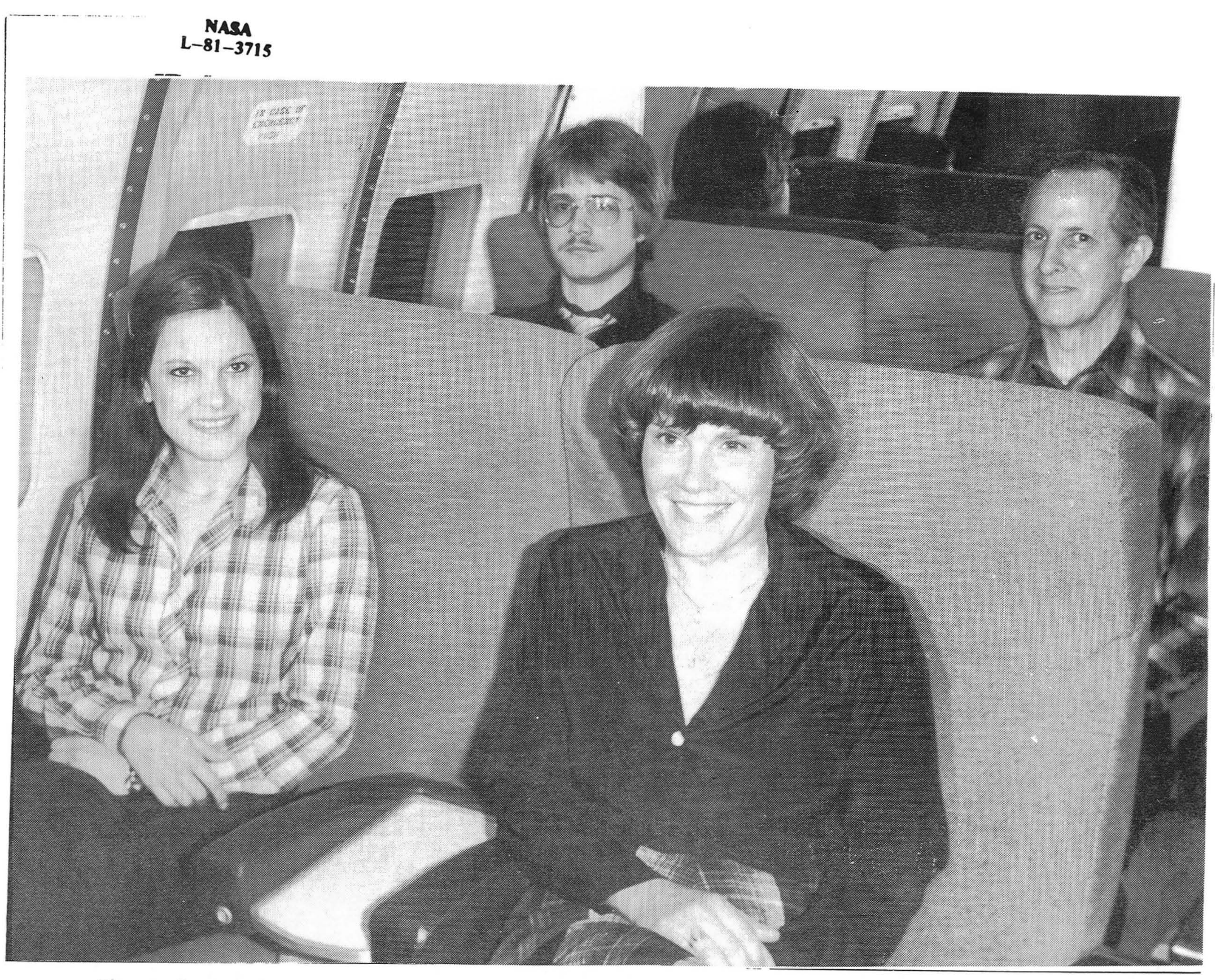

Figure 1.- Photograph of the passenger ride quality apparatus with the front bulkhead removed. 


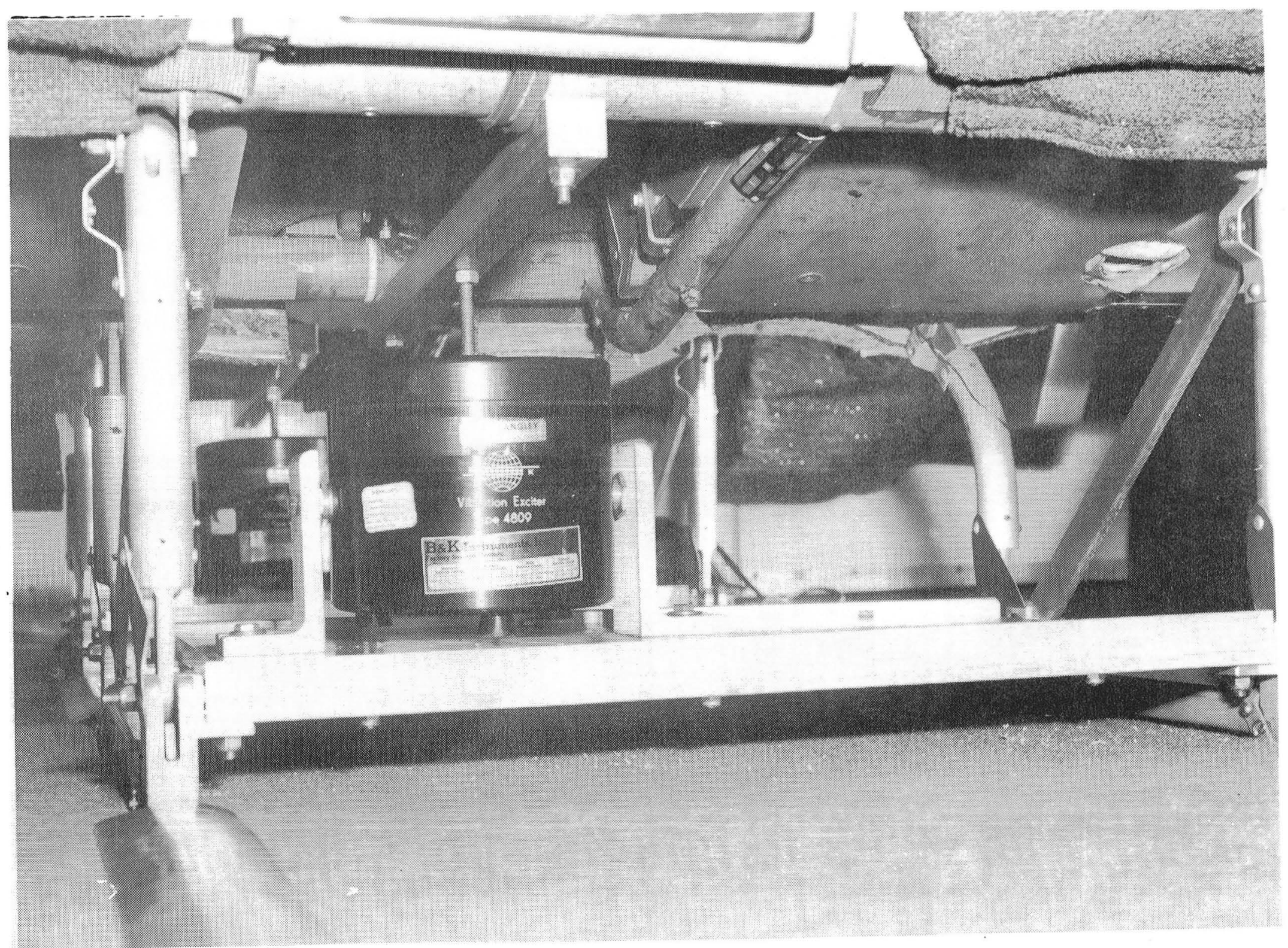

Figure 2.- Photograph of the shaker installation under the seats. 
Subject Number

Session Number
Date

Age a.m. p.m. Sex

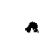

Ride Number Annoyance
Maximum

Annoyance

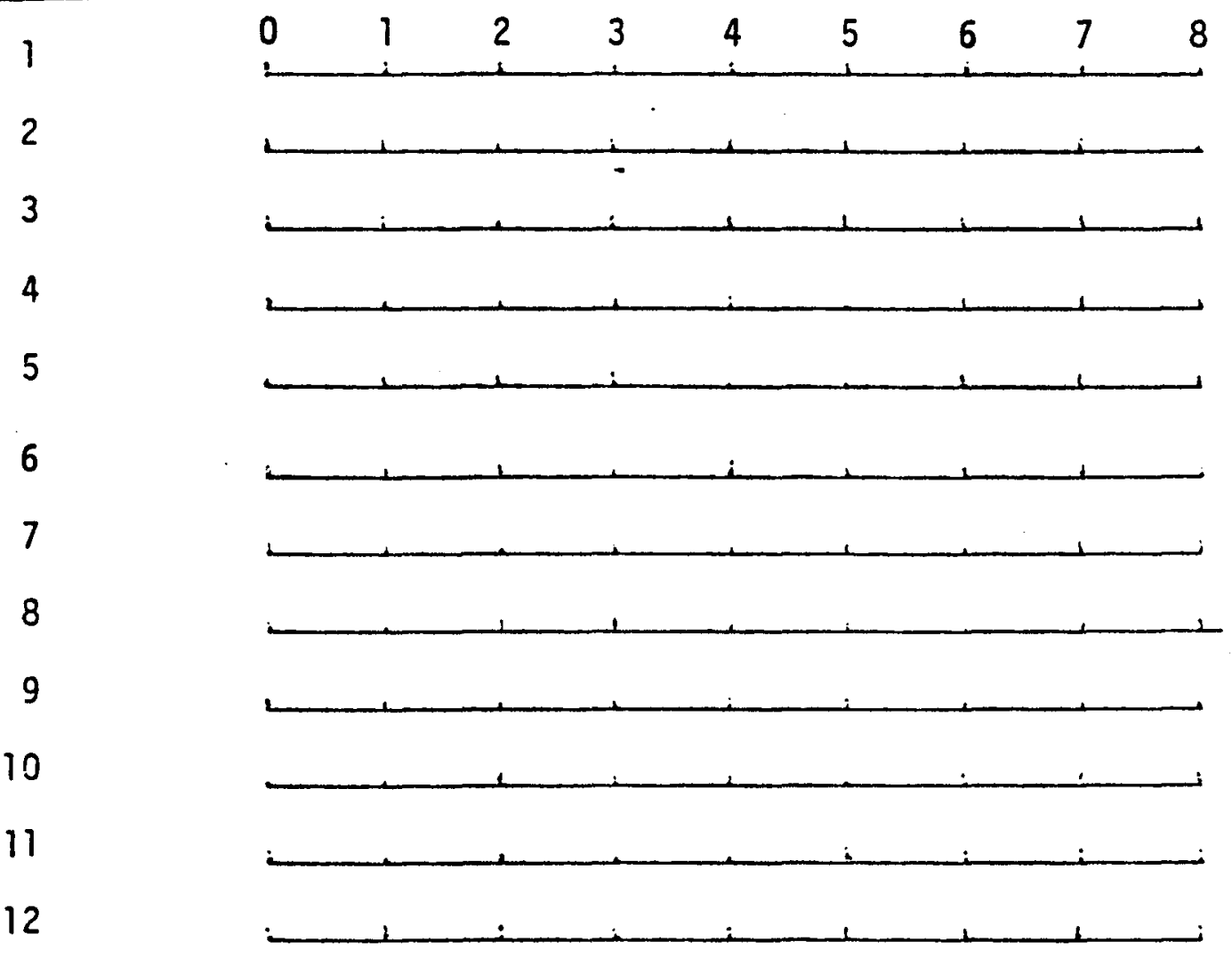

13

14

15

16

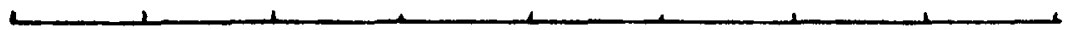

Figure 3.- Sample score sheet. 

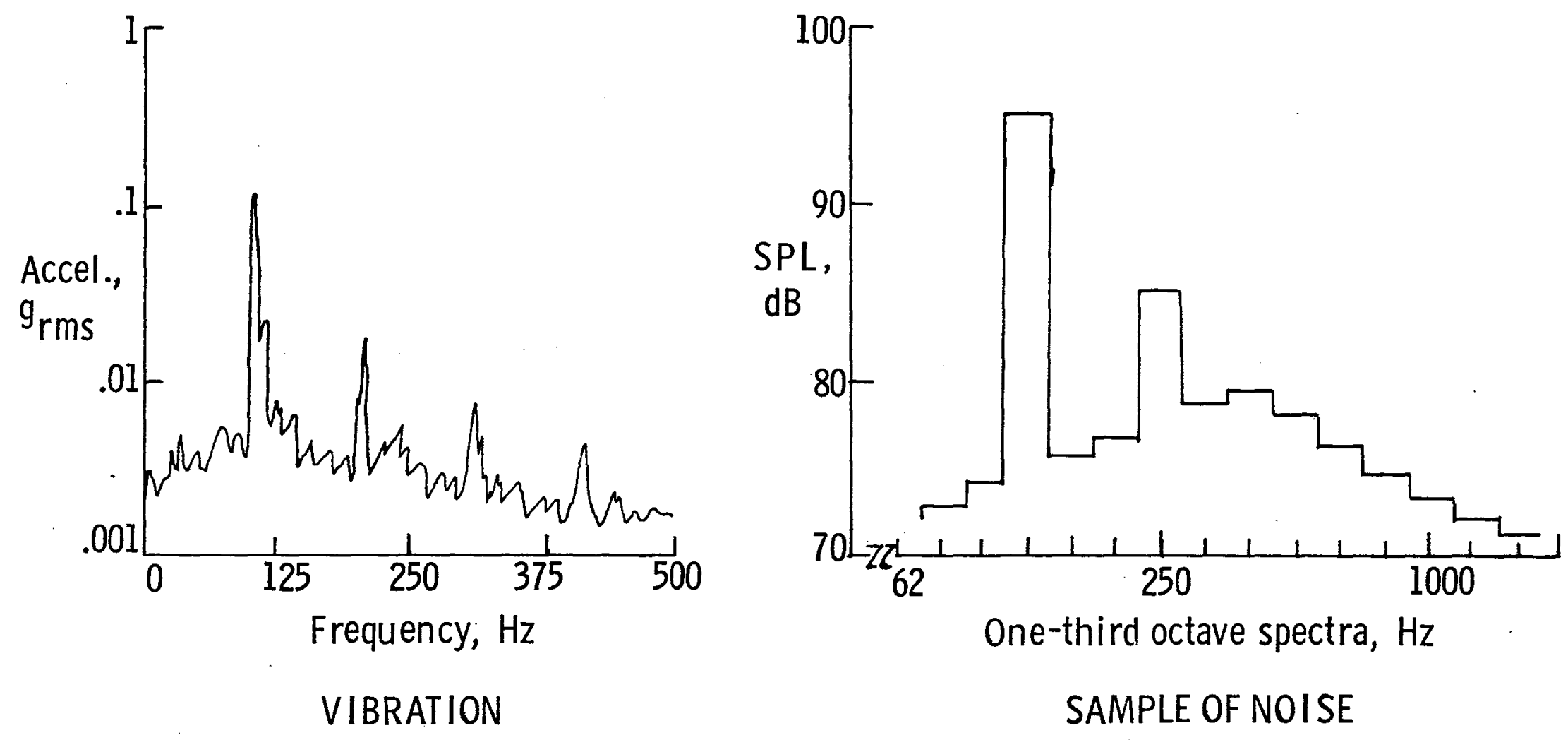

Figure 4. - Examples of the spectra of the cabin interior noise and seat-arm vibration. 


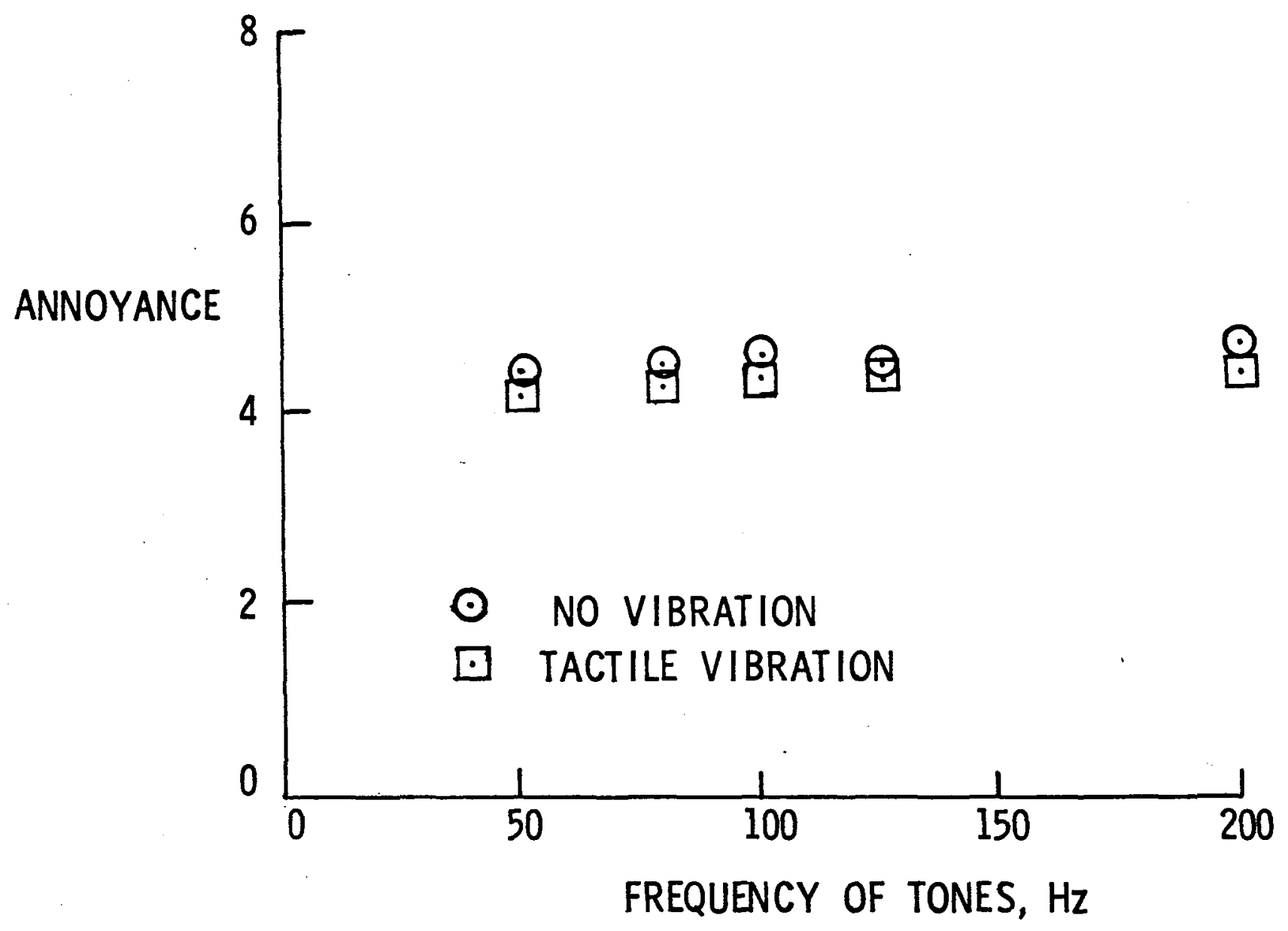

Figure 5. - Annoyance as a function of fundamental frequency of tones to show the effect of tactile vibration. 


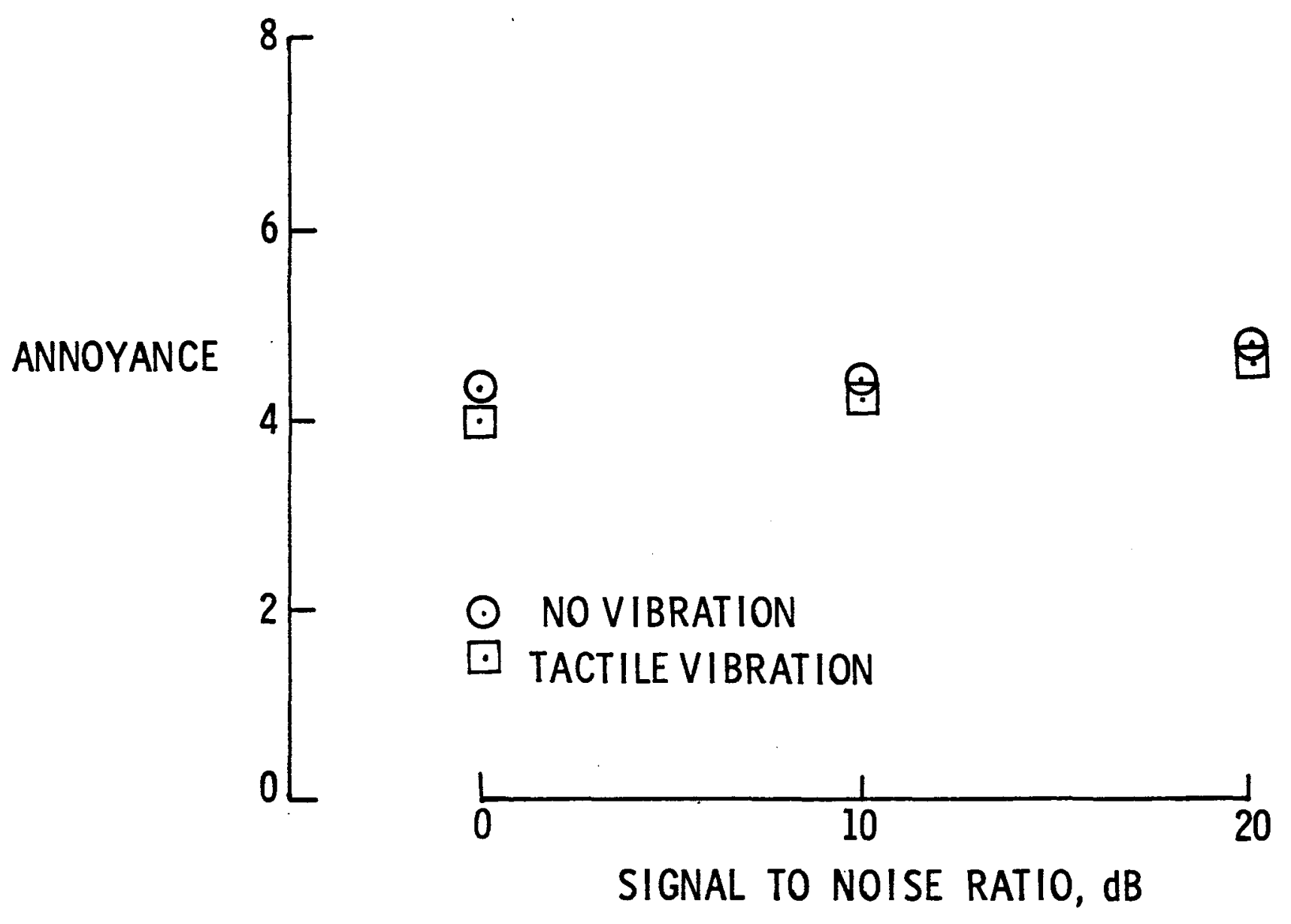

Figure 6. - Annoyance as a function of signal to noise ratio to show the effect of tactile vibration. 


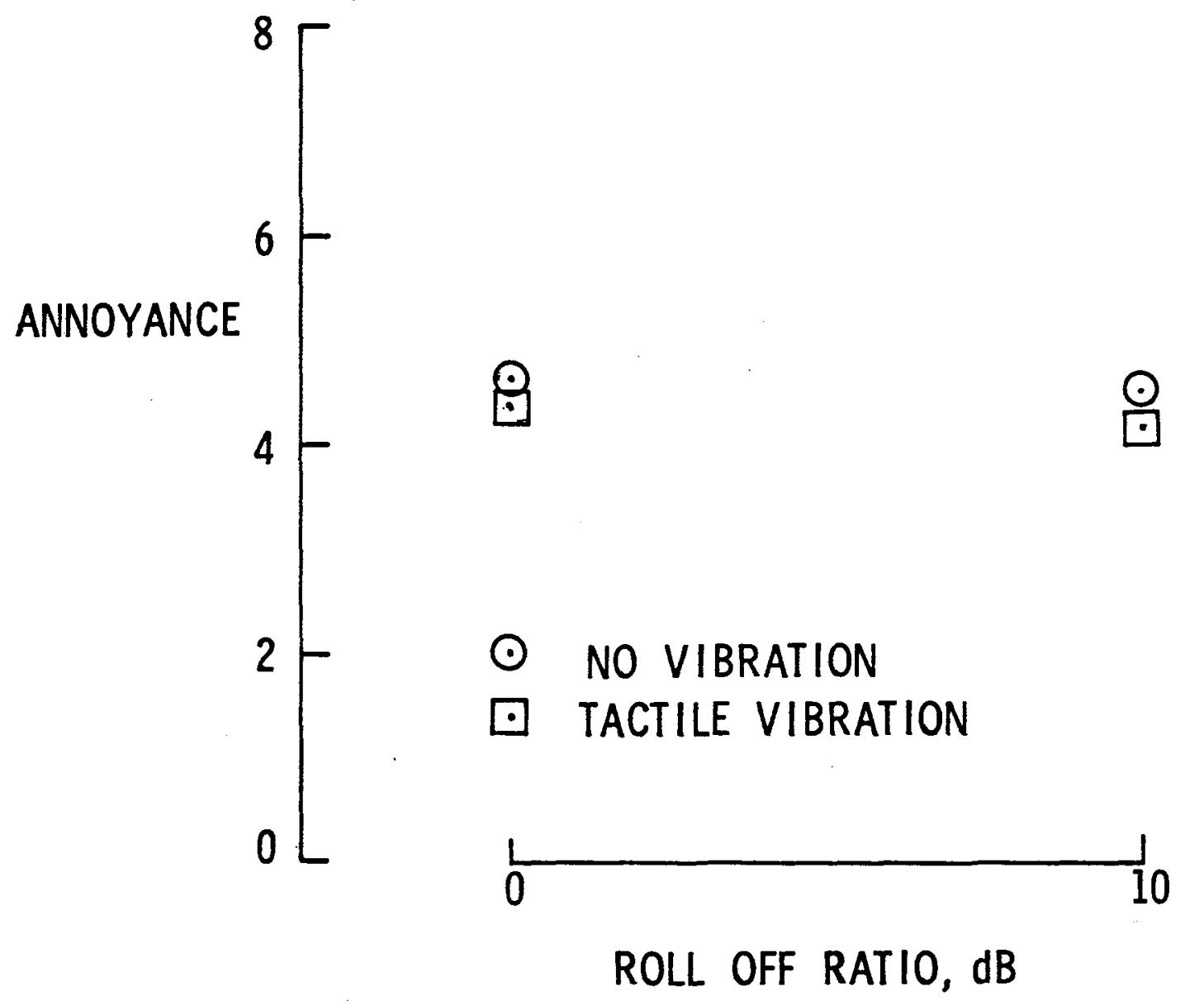

Figure 7. - Annoyance as a function of roll off ratio to show the effect of tactile vibration. 


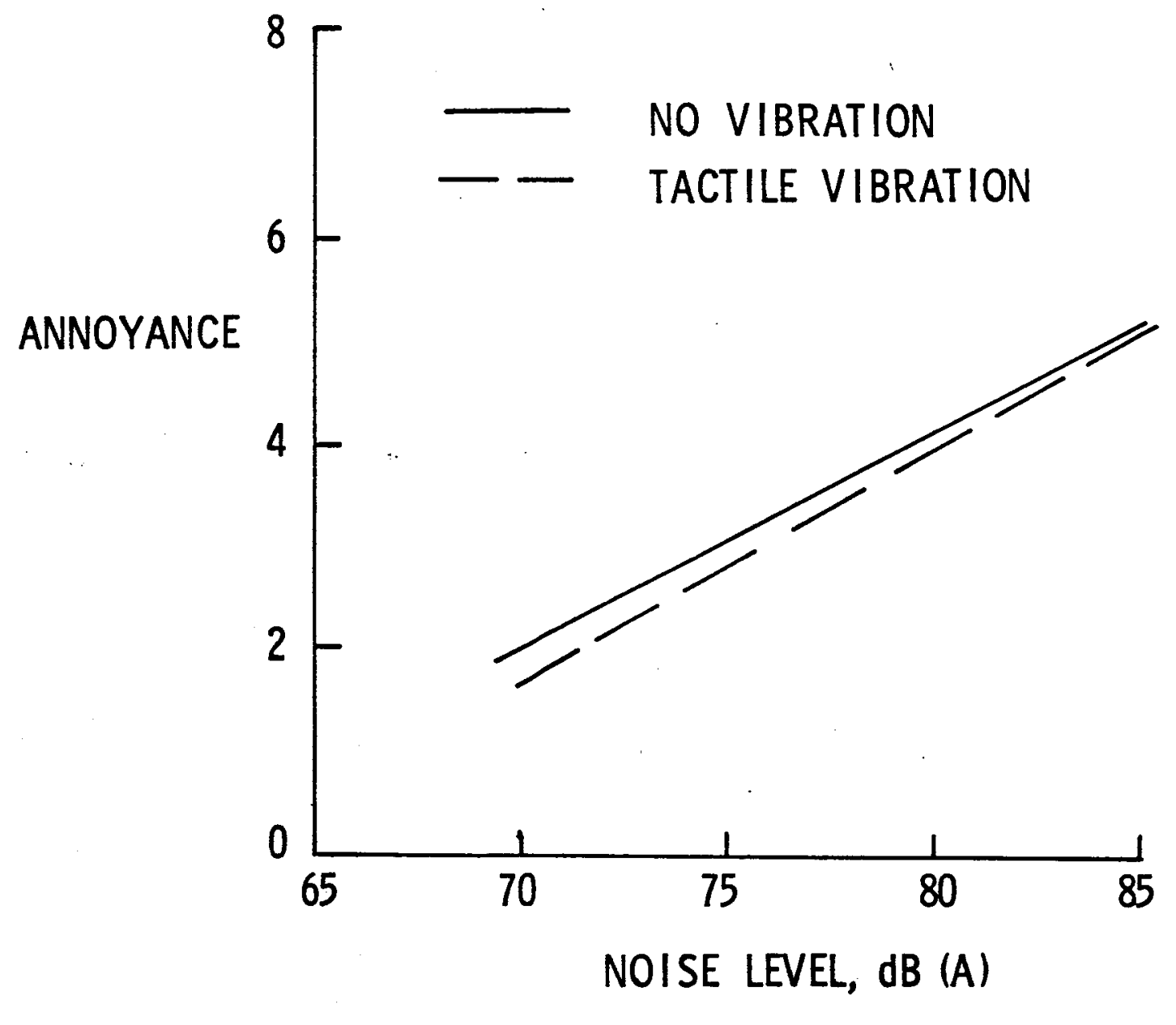

Figu re 8. - Annoyance as a function of " $A$ " weighted noise level to show the effect of tactile vibration. 


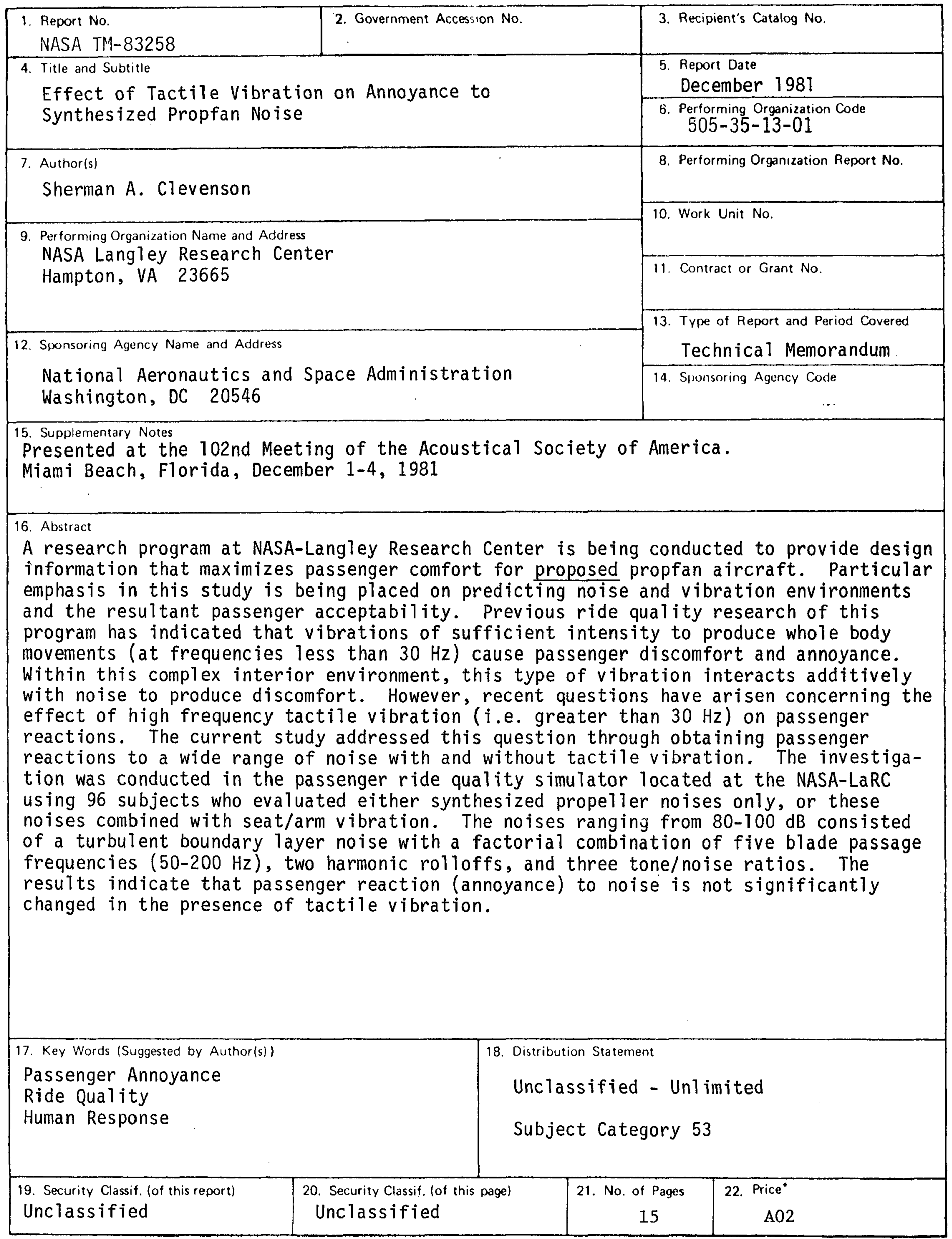

\footnotetext{
* For sale by the National Technical Information Service, Springfield. Virginia 22161
} 


